\title{
Suture fixation of dislocated endothelial grafts
}

\begin{abstract}
Purpose To share the experience of suturing the displaced endothelial grafts in three patients who developed this complication following a posterior endothelial keratoplasty. Methods Prospective evaluation of three patients who underwent surgical revision by suture fixation for dislocated endothelial graft. The surgeries were performed immediately after noting the complication during the postop visits and were followed up for 6 months. Assessments at follow-up visits include visual acuity, pachymetry, endothelial cell count, topography, and corneal slit-lamp digital photography.

Results No significant postoperative complications were encountered in any of the three eyes after graft suturing. The vision was worse than hand movements in all, following the graft dislocation and this improved to an average of 20/50 at 6-month post-op. In two of the three eyes the endothelial cell count reduced from 2600 cells preoperatively to 1251 and 988 cells, respectively. Mean topographic astigmatism after the suture removal was $2.4 \mathrm{D}$. Conclusion Suture fixation of early endothelial graft dislocation seems to be a good alternative in dislocated endothelial discs as it provides a good refractive and visual outcome, instead of converting it to a standard full-thickness penetrating keratoplasty.

Eye (2008) 22, 718-721; doi:10.1038/sj.eye.6703000; published online 7 March 2008
\end{abstract}

Keywords: posterior endothelial keratoplasty; descemet-stripping endothelial keratoplasty; corneal graft; graft dislocation; endothelial graft suturing

12 September 2007

Published online: 7 March

2008

Presented as a poster at the Royal College of

Ophthalmology Congress, 2006.

\section{Introduction}

Endothelial failure is routinely treated by penetrating keratoplasty (PK), with established efficacy but slow visual rehabilitation. Posterior endothelial keratoplasty replaces a lamella of endothelium/Decemet's membrane, and offers rapid visual recovery by retention of host stroma.

Although longer-term follow-ups are not yet known as this is a relatively new technique, early complications such as graft dislocation, graft rejection, and endothelial failure have been reported. Early graft slippage or dislocation is one of the most common postoperative challenges and requires revision surgery. ${ }^{1} \mathrm{We}$ describe three cases of recurrent donor lamellar dislocation into the anterior chamber, successfully treated by air bubble repositioning and suture fixation. The surgeries were performed immediately after noting the complication during the post-op visits and were followed up for 6 months. Assessments at follow-up visits included visual acuity, pachymetry, endothelial cell count, topography, and corneal slit-lamp digital photography.

\section{Surgical procedure}

All the patients underwent an uneventful/ uncomplicated Descemet-stripping endothelial keratoplasty. The donor button was prepared by manual stromal dissection in an air-filled artificial chamber (Melles' technique).

Descemet's stripping was performed aiming for a diameter of approximately $9 \mathrm{~mm}$. A $9-\mathrm{mm}-$ folded donor disc was placed through a $5-\mathrm{mm}$ scleral incision. Small amount of viscoelastic was used, only on the endothelial side of the disc before folding. Air was injected into the AC to help unfold the donor tissue and oppose it against the recipient cornea. When found dislocated, all patients underwent repositioning of the graft on the first postoperative day under the operating microscope. Sterile air through the paracentesis was used to refloat the graft into position. All the three eyes described here were dislocated again after refloating and were then sutured in place as described below. 


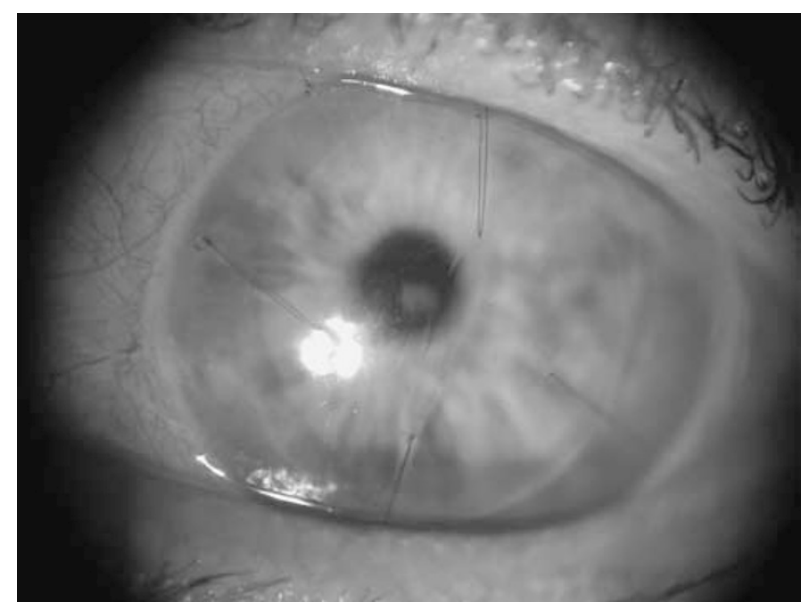

Figure 1 Sutured endothelial graft.

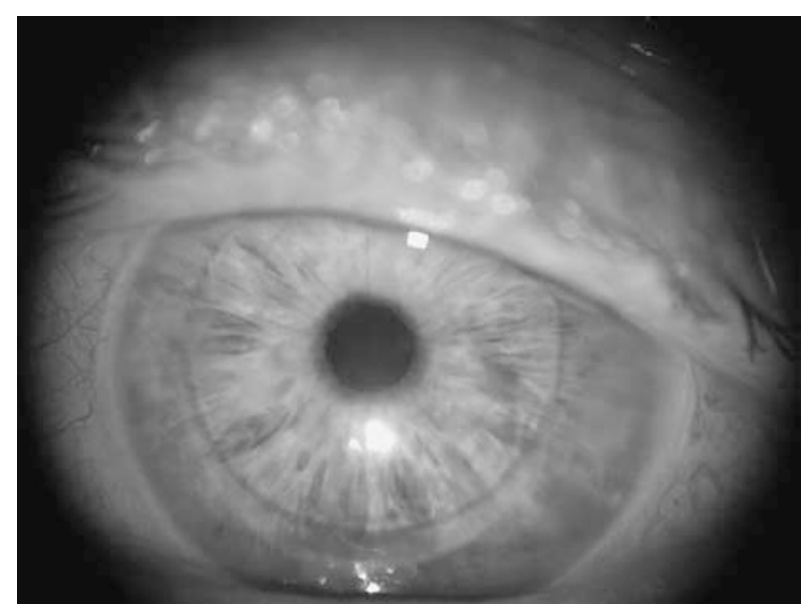

Figure 2 After suture removal.

All the surgeries were performed by a single surgeon (ML) in the first postoperative week immediately after noting the complication (recurrent dislocation of the graft). A paracentesis was made and the donor disc centred using forceps or a sinskey hook. It was then floated into apposition with the cornea on a sterile air bubble. 10-0 nylon sutures (Alcon, 665567M, UK) were then placed, passing from the peripheral cornea inwards, and then passing through the donor tissue, continuing through the full thickness of the cornea. The sutures were tied on adjustable sutures and tension was adjusted once all sutures were in place. Four to six sutures were placed until the graft appeared to be securing with good adhesion formed in all $360^{\circ}$ of the edge of the interface. Anterior chamber was reformed at this stage again with air to provide enough tamponade to avoid Descemet's folds, prior to tying-off. The sutures were then rotated to bury the knot. It was necessary to ensure that the last movement of the suture was a rotation away from the graft, thus pulling the graft out towards the peripheral

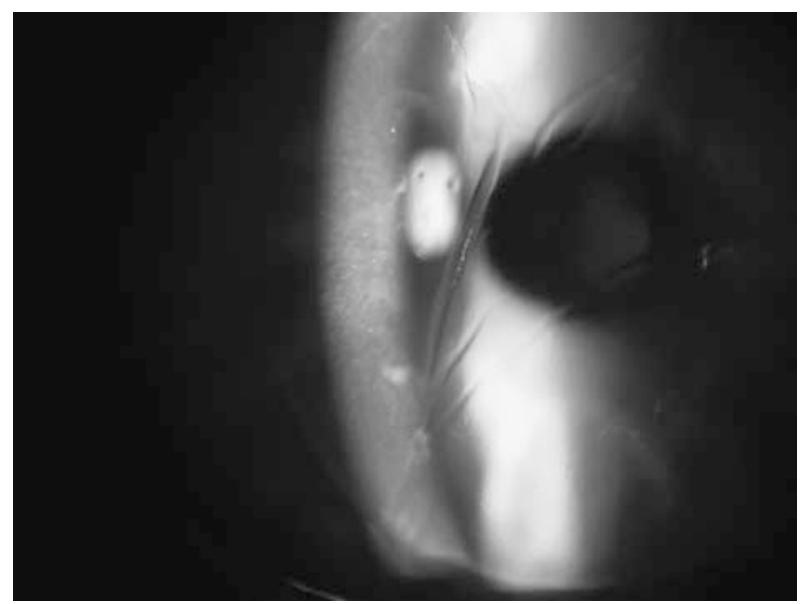

Figure 3 Descemet's folds after suture removal.

cornea. In two eyes, four sutures were placed; in one eye, six were placed. The procedure took about $15 \mathrm{~min}$ and involved topical anaesthesia under an operating microscope. Sutures were removed 6 weeks after surgery (Figures 1 and 2 - Pre- and post-suture removal).

\section{Results}

The initial pathology in all the three patients was Fuch's endothelial dystrophy.

The patient ages at surgery were 76, 79, and 60, respectively; all three were left eyes.

No postoperative complications occurred. Clinically, the graft appeared clear under the slit lamp in all three cases but one eye had Descemet's folds at 3 months follow-up (Figure 3). The vision was worse than hand movement in all following graft dislocation and this improved to an average of 20/50 at 6 months post-op. At 6 months follow-up, the average central corneal thickness was $595 \mu \mathrm{m}$ compared to preoperative average of $685 \mu \mathrm{m}$ (measured prior to initial lamellar graft procedure). In one of the three cases endothelial cell count was not possible, but in other two cases the cell count reduced to 1251 and 988 cells compared to a preoperative cell count of 2600 in both the donor corneas. This represents a loss of $55.1 \%$ during the surgery and postoperative period. The average age of the donor tissue was 80.3 years. Mean topographic astigmatism after the suture removal was 2.4 D. The visual acuities, pachymetry, astigmatism, and endothelial cell count at 6 months are presented in Table 1.

\section{Discussion}

Posterior keratoplasty is a new surgical technique that may be valuable in treating patients with corneal decompensation secondary to endothelial dysfunction. 
Table 1 Showing the visual acuity, pachymetry, astigmatism, donor graft age and endothelial cell count at 6 months after suturing the graft

\begin{tabular}{lccccc}
\hline & $\begin{array}{c}\text { Best-corrected VA } \\
\text { pre-op -post-op }\end{array}$ & $\begin{array}{c}\text { Pachymentry at } \\
\text { pre-op-post-op }\end{array}$ & $\begin{array}{c}\text { Astigmatism using } \\
\text { topography }\end{array}$ & $\begin{array}{c}\text { Endothelial cell count } \\
\text { pre-op -post-op }\end{array}$ & $\begin{array}{c}\text { Donor graft } \\
\text { age }\end{array}$ \\
\hline Patient 1 & $20 / 80-20 / 60$ & $668-639$ & 1.75 & $2600-1251$ & 86 \\
Patient 2 & $20 / 60-20 / 40$ & $663-517$ & 3.5 & $2600-$ unattainable & 81 \\
Patient 3 & $20 / 80-20 / 60$ & $724-630$ & 2 & $2600-988$ & 74 \\
\hline
\end{tabular}

It selectively replaces diseased endothelium with healthy donor tissue. Over the past several years, this surgery has undergone extensive modifications and the published reports on several small series have suggested distinct advantages over standard full-thickness PK. ${ }^{1-5}$ The advantages include improved surface topography with the elimination or reduction of irregular astigmatism, improved predictability of postoperative-corneal refractive power with better intraocular-lens calculation, and retention of good endothelial cell densities and function. ${ }^{6-8}$ All these advantages have resulted in faster visual rehabilitation for the patient. ${ }^{9}$

However, every surgery has its own risks and complications. Graft dislocation, rejection, graft failure, glaucoma, and cataract have all been noted. ${ }^{1,10}$ Successful Posterior lamellar keratoplasty requires the donor disc to self-adhere during and after surgery. Possible causes for dislocation are thought to be:

(1) Residual viscoelastic material in the interface

(2) Improper position of the donor disc peripheral edge, such that it lies posterior to the edge tissue of the recipient bed

(3) Delayed endothelial pump function

(4) Primary graft failure

(5) Donor disc placed upside down. ${ }^{10}$

Even after meticulous attention to the abovementioned factors dislocation can still happen, and may have adverse effects on the graft: Terry and Ousley ${ }^{10}$ have reported a significantly lower average endothelial-cell count at 6-month post-op (1534 \pm 366 cells $/ \mathrm{mm}^{2}$ ) in eyes that had donor dislocation than in eyes that did not have dislocation $(2166 \pm 411$ cells $/ \mathrm{mm}^{2}$ ). One way of managing this complication postoperatively is to reposition the graft under air, described by Terry, ${ }^{10}$ but one or more causes of dislocation may persist and therefore dislocation recurs once the air bubble has disappeared. Other options to manage dislocations are to replace the endothelial graft with a new one or to covert to a full-thickness PK.

All our three patients underwent an uneventful/ uncomplicated Descemet-stripping endothelial keratoplasty (DSEK). The donor disc was manually dissected using an artificial anterior chamber approximately to the same size as the diameter of the resection of the recipient's stroma. We did not encounter any problems during the dissection of donor or recipient cornea. This was later confirmed by normal corneal pachymetry postoperatively in all three cases. All the dislocations were noted immediately after the surgery and were repositioned on the first postoperative day under air but were found dislocated again the next day when the air bubble, which was used to hold the graft in place, disappeared. We suspect a delayed endothelial pump function due to unknown reason as the cause for recurrent dislocation. With the patients' informed and written consent, we sutured the dislocated graft in place until the graft appeared to be securing with good adhesion formed in all 360 degrees of the edge of the interface. This required four sutures in two of our patients and six in the remaining one patient. The aim of the suturing was to engage the peripheral edge of the donor tissue to support it in place until the endothelial pump function took over. The sutures were removed at 6 weeks after surgery.

In the Price et al. ${ }^{1}$ series, donor detachment rate of $50 \%$ was noted in the first 10 cases that reduced to $13 \%$ in the next 126 cases and 3\% in the final 64 cases. ${ }^{1}$ The reduction in dislocation rate was attributed to the surgeons' learning curve and also to various other techniques to remove the fluid from the donor-recipient graft interface. In the Terry and Ousley ${ }^{10}$ series, of 98 eyes that under went DLEK (deep lamellar endothelial keratoplasty), four patients were found to have dislocation of the graft on the first postoperative day. Mean best spectacle-corrected vision in patients who had graft dislocation was $20 / 46$ and the average endothelialcell count was $1534 \pm 366,6$ months after surgery. In various other published series, the best-corrected vision following lamellar endothelial replacement ranges from $20 / 40$ to $20 / 50$, which compares well with our average of $20 / 50.4,7,11$

We found the postoperative endothelial-cell loss was $55.1 \%$ compared to preoperative donor tissue. Melles et $a l^{11}$ found $43.1 \%$ endothelial-cell loss at 6 months in one series following a $5 \mathrm{~mm}$ incision posterior lamellar keratoplasty. However in another series, Terry and Ousley $^{7}$ have reported $24.1 \%$ endothelial loss at 6 months post-op. In both these series, endothelial count was from 
non-dislocated grafts. The increased endothelial loss seen in our patients may be

(1) A cause of dislocation due to inadequate endothelial pump

(2) A result of recurrent dislocation (all eyes were sutured after a failed repositioning with air bubble)

(3) A result of tissue damage from donor manipulation during repositioning and suturing

We recommend suturing the graft after the first reposition for dislocation as to avoid repeated dislocation and further endothelial loss. As we do not have any comparative data for endothelial loss with repostponing under air bubble and primary suturing after first dislocation, we could not recommend this procedure to treat the initial dislocation yet.

In Melles et al' series ${ }^{4}$, of seven eyes following PELK (Posterior endothelial lamellar keratoplasty) the average postoperative astigmatism was reported as $1.54 \mathrm{D}$.

Looking at stability of topography in 20 eyes following DELK, Ousley and Terry ${ }^{8}$ found the average topographic astigmatism at 1 year to be $2.3 \pm 1.1 \mathrm{D}$ and 2 year to be $2.4 \pm 1.1 \mathrm{D}$. This compares well with our series of three cases with an average topographic astigmatism of $2.4 \mathrm{D}$ after suture removal. The sutures just held the endothelium in place during the initial few weeks and did not have any adverse effect on astigmatism immediate post-op and also after suture removal.

One of the patients developed Descemet's folds, which remained even after suture removal (Figure 3).

Fortunately, it did not affect the visual outcome significantly. Corneal graft folds have been noted before following posterior endothelial keratoplasty even without suturing. ${ }^{12}$ Suture traction could have directly contributed to the endothelial folds but improper centration within the recipient bed or an inadvertently undersized recipient bed diameter could also have been a reason for it. Using penetrating suturing to the endothelial graft could also increase the chance of endophthalmitis and epithelial ingrowths, especially if the sutures become loose prior to removal. We did not encounter any such complications in our very small case series.

By suturing the dislocated endothelial graft we believe that we avoided additional major surgery for the patient, retained the advantage of rapid postoperative recovery, and prevented wastage of corneal donor tissue. The patients still benefited from early visual rehabilitation of the posterior endothelial keratoplasty as apposed to a situation of having to covert to a standard PK. We only had to suture three eyes until now and there have been no failures in this technique. To our knowledge there is no published data on suture fixation of endothelial grafts.

\section{References}

1 Price Jr FW, Price MO. Descemet's stripping with endothelial keratoplasty in 200 eyes: early challenges and techniques to enhance donor adherence. J Cataract Refract Surg 2006; 32(3): 411-418.

2 Terry MA. A new approach for endothelial transplantation: deep lamellar endothelial keratoplasty. Int Ophthalmol Clin 2003; 43(3): 183-193.

3 Melles GR, Eggink FA, Lander F, Pels E, Rietveld FJ, Beekhuis WH et al. A surgical technique for posterior lamellar keratoplasty. Cornea 1998; 17(6): 618-626.

4 Melles GR, Lander F, van Dooren BT, Pels E, Beekhuis WH. Preliminary clinical results of posterior lamellar keratoplasty through a sclerocorneal pocket incision. Ophthalmology 2000; 107(10): 1850-1856.

5 Melles GR, Lander F, Nieuwendaal C. Sutureless, posterior lamellar keratoplasty: a case report of a modified technique. Cornea 2002; 21(3): 325-327.

6 Terry MA, Ousley PJ. In pursuit of emmetropia: spherical equivalent refraction results with deep lamellar endothelial keratoplasty (DLEK). Cornea 2003; 22(7): 619-626.

7 Terry MA, Ousley PJ. Small-incision deep lamellar endothelial keratoplasty (DLEK): six-month results in the first prospective clinical study. Cornea 2005; 24(1): 59-65.

8 Ousley PJ, Terry MA. Stability of vision, topography, and endothelial cell density from 1 year to 2 years after deep lamellar endothelial keratoplasty surgery. Ophthalmology 2005; 112(1): 50-57.

9 Terry MA, Ousley PJ. Rapid visual rehabilitation after endothelial transplants with deep lamellar endothelial keratoplasty (DLEK). Cornea 2004; 23(2): 143-153.

10 Terry MA, Ousley PJ. Deep lamellar endothelial keratoplasty: early complications and their management. Cornea 2006; 25(1): 37-43.

11 Van Dooren B, Mulder PG, Nieuwendaal CP, Beekhuis WH, Melles GR. Endothelial cell density after posterior lamellar keratoplasty (Melles' techniques): 3 years follow-up. Am J Ophthalmol 2004; 138(2): 211-217.

12 Faia LJ, Baratz KH, Bourne WM. Corneal graft folds: a complication of deep lamellar endothelial keratoplasty. Arch Ophthalmol 2006; 124: 593-595. 